\title{
Numerical stabilisation of motion integration
}

\author{
D. S. Holloway ${ }^{1}$
}

(Received 18 July 2006; revised 06 July 2007)

\begin{abstract}
Explicit time stepping of initial value problems may be unstable when the highest derivative cannot be isolated. The example of time domain ship motions is studied, in which instability arises from feedback from implied acceleration terms in the hydrodynamic force associated with the 'added mass' of the water surrounding the hull. By combining the acceleration computed from the hydrodynamic forces with one obtained by extrapolating the recent motion history a stable and accurate solution is obtained. The problem is studied using a simpler approximation after demonstrating its equivalence. The proposed technique may be applied to any problem exhibiting the same instability.
\end{abstract}

\section{Contents}

1 Introduction and source of instability

See http://anziamj.austms.org.au/ojs/index.php/ANZIAMJ/article/view/51 for this article, (c) Austral. Mathematical Soc. 2007. Published July 16, 2007. ISSN 1446-8735 
2 Existing solution methods

C251

3 Method of stabilisation

C252

4 Constant coefficient simulation

C254

5 Limiting $\mu$ for stability

C257

6 Influence of $\mu$ on accuracy

C259

7 Conclusion

C261

References

C262

\section{Introduction and source of instability}

While frequency domain ship motion computations are routinely performed it is becoming increasingly desirable to obtain efficient time domain solutions. Time domain solutions are necessary to simulate nonlinear phenomena such as motions in heavy seas or wave impact, but are inherently unstable. Often an explicit time stepping method is desired, and all the cited stabilisation techniques fail in these circumstances. A simple technique overcoming these obstacles is presented and discussed.

Ship motions in waves involve equations essentially of the form $F(t)=$ $m \ddot{x}$, where $x$ represents a generic motion variable in any of the ship's degrees of freedom and $F$ the forces causing that motion. $F$ is calculated by integrating the pressure distribution over the hull surface, where local pressures take the (linearised) form $p=-\rho \partial \phi / \partial t$. In turn, the velocity potential $\phi$ is obtained by solving Laplace's equation subject to some boundary conditions, including $\partial \phi / \partial n=\mathbf{V} \cdot \mathbf{n}$ on the ship's hull. Finally the local hull velocity $\mathbf{V}$ depends on $\dot{x}$. Thus $F$ implicitly depends on $\ddot{x}$, and it is well 
known that numerical solutions of differential equations are unstable if the highest derivative cannot be isolated.

To identify more specifically the source of instability consider the equation of motion for a single degree of freedom in its usual frequency domain form,

$$
(m+A) \ddot{x}+B \dot{x}+C x=F_{w},
$$

where $F_{w}$ represents the incident and diffracted wave forces, which are independent of $x$, while $A, B, C$ and $m$ are respectively the 'added mass' (inertia of the water surrounding the hull), 'damping coefficient' (representing the energy transported in the radiated waves), hydrostatic stiffness coefficient and ship mass. The corresponding time domain form is

$$
m \ddot{x}=F_{w}-(A \ddot{x}+B \dot{x}+C x) .
$$

We must recognise that $A, B$ and $C$ are generally weakly time (or more strictly frequency) dependent, but if we integrate with a small time step we can treat them as constants over each time step.

In a time domain solution the right hand side of (2) as a whole is known but it is not decomposed as in (2). Suppose there is a small error $\epsilon$ in $\ddot{x}$, which will be apparent only as an error of $-\epsilon A$ in the total force at that time instant. At each time step the error will be amplified by $-\alpha A / m$ ( $\alpha$ being introduced to account for additional effects such as influence of the particular time stepping algorithm used and the dependence of $A$ on $\ddot{x}$ ). This amplification factor must have an absolute value less than 1 for an integration method to be stable.

\section{Existing solution methods}

It is common practice in analysing ship motion, even in time domain simulations, to avoid the problem by calculating radiation and diffraction forces 
separately. This is not only inefficient but it does not permit the study of nonlinearities as it relies on superposition. Beck et al. [1] offer an alternative solution. They argue that $\partial \phi / \partial t$ is critical to the calculation of hydrodynamic forces and must be obtained by a backward difference which gives a poor estimate of the derivative leading to the observed instability. However, $\partial \phi / \partial t$ satisfies the same partial differential equation as $\phi$ so can be computed directly avoiding the backward difference. The same singularity may be used for both $\phi$ and $\partial \phi / \partial t$, avoiding the need to create and invert another influence matrix. Unlike $\phi$, which only requires body velocities in its boundary condition, $\partial \phi / \partial t$ requires body accelerations. They assume an acceleration, calculate $\partial \phi / \partial t$ and hence $F$, and from $F$ calculate the new acceleration. Stability is achieved by iterating the process until the assumed and calculated accelerations agree to within a given tolerance.

The method of Beck et al. [1] is suitable when most of the computational effort is in assembling and inverting the influence matrix. However, I, for example, used a Green function method for evaluating hydrodynamic forces [2], which, even with no iteration, requires twice as many calculations.

A different approach, formally analysed for linear problems by Kring and Sclavounos [4], involves separating the fluid velocity potential into an instantaneous (infinite frequency) component and a memory component (impulse response). Thus in effect $A$ in (1) is given a value $A_{0}$ say, corresponding to infinite frequency, while the vessel's impulse response is embodied into the force $F_{w}$. Again this approach was deemed unsuitable as $A_{0}$ is difficult to separate and time consuming to compute.

\section{Method of stabilisation}

A method was sought that was stable, insensitive to initial conditions, gave accurate results, required a single evaluation of the right hand side of (2), and could be used with equal time step sizes without the need for iteration 
or evaluation of intermediate values. A technique was developed similar to that of Kring and Sclavounos [4] in that it separates the instantaneous added mass (the source of the instability) from the hydrodynamic force. However a major difference is that it was found to be sufficient to estimate $A_{0}$ within a broad range and that it is therefore not necessary to decompose $F_{w}$ to compute a precise $A_{0}$.

The technique employed involves setting the acceleration at each time step at a weighted average of the value calculated from the hydrodynamic forces and a value extrapolated from the recent velocity history. Although neither acceleration on its own leads to stable or accurate motion integration, the two have errors of opposite sign and, if combined in the right proportions, give a stable and accurate solution.

Starting with (2), remove the source of instability by adding $A \ddot{x}$ to both sides. These are both unknown so we estimate $A \approx A_{e}$ and $x \approx x_{e}$, hence

$$
\ddot{x}\left(m+A_{e}\right)=F+A_{e} \ddot{x} \approx F+A_{e} \ddot{x}_{e},
$$

from which

$$
\ddot{x} \approx \mu \ddot{x}_{c}+(1-\mu) \ddot{x}_{e}
$$

where $\ddot{x}_{c}=F / m$ is the calculated acceleration, $\mu=m /\left(m+A_{e}\right)$ is a coefficient to be determined, and $x_{e}$ is estimated from the immediately prior velocity history, for example by backwards difference.

To determine when this will stabilise the solution, suppose the error $\epsilon$ at any given time step is amplified by $-\rho_{c}$ using $\ddot{x}_{c}=F / m(\mu=1)$ and by $+\rho_{e}$ using $\ddot{x}_{e}(\mu=0)$ where $\rho_{c}, \rho_{e}>1$ or the problem is trivially solved. It follows from (4) that stability requires $\left|-\rho_{c}+\rho_{e}(1-\mu)\right|<1$, hence

$$
\frac{\rho_{e}-1}{\rho_{e}+\rho_{c}}<\mu<\frac{\rho_{e}+1}{\rho_{e}+\rho_{c}} .
$$

Thus a stable solution is obtained for a range of $\mu$. It is shown below that the limiting values of $\mu$ for stability are sharp, the deterioration of the solution 
beyond the range of valid $\mu$ values is obvious, and acceptable accuracy is maintained right up to the limiting $\mu$, so there is little danger of accepting an erroneous solution.

Finally, we require an estimate $\ddot{x}_{e}$ for which $\rho_{e}>1$. We use a second order backward difference approximation to the velocity derivative:

$$
\ddot{x}_{e}(t)=\frac{3 \dot{x}(t)-4 \dot{x}(t-\Delta t)+\dot{x}(t-2 \Delta t)}{2 \Delta t} .
$$

Having stabilised $\ddot{x}$ using (4) any standard time stepping method may be used to integrate it. Here we use a Taylor method with extrapolated third derivative for slightly improved accuracy:

$$
\begin{aligned}
\dddot{x}(t) & =\frac{3 \ddot{x}(t)-4 \ddot{x}(t-\Delta t)+\ddot{x}(t-2 \Delta t)}{2 \Delta t}, \\
\dot{x}(t+\Delta t) & =\dot{x}(t)+\Delta t \ddot{x}(t)+\frac{\Delta t^{2}}{2} \dddot{x}(t), \\
x(t+\Delta t) & =x(t)+\Delta t \dot{x}(t)+\frac{\Delta t^{2}}{2} \ddot{x}(t)+\frac{\Delta t^{3}}{3 !} \dddot{x}(t) .
\end{aligned}
$$

It follows from (8) that (6) will give $\rho_{e} \approx 1.5$.

\section{Constant coefficient simulation}

Solution of the full ship hydrodynamic problem is highly computationally intensive but for the purposes of studying the above stabilisation algorithm it is sufficient to represent $F$ as

$$
F=F_{w}-(A \ddot{x}+B \dot{x}+C x),
$$

where $F_{w}$ may be arbitrarily specified, $A, B$ and $C$ are treated as constants, and $\ddot{x}$ on the right hand side is taken as the estimated value, $\ddot{x}_{e}$ from equation (6). 


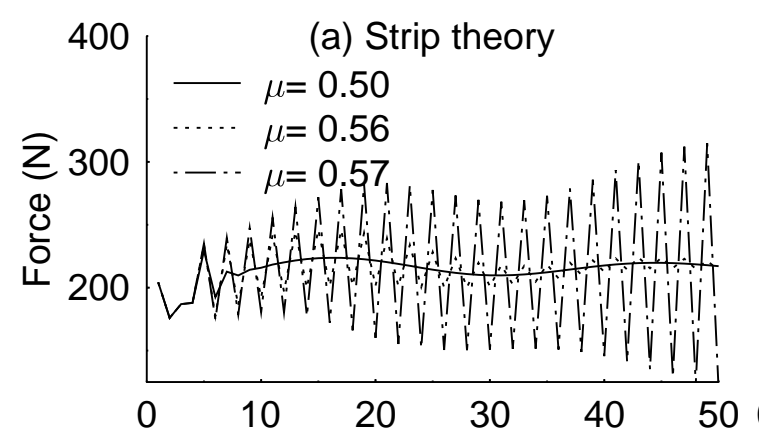

(b) Constant coefficient simulation

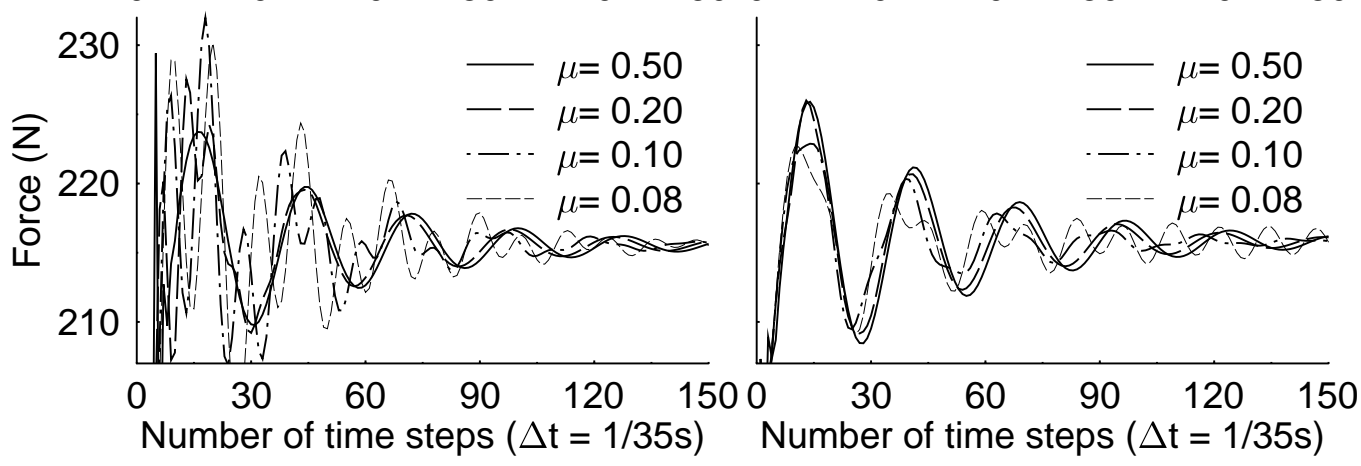

FigURE 1: Behaviour of the numerical integration. Upper half: $\mu$ near the upper stability limit. Lower half: $\mu$ near the lower stability limit. 
Validity of this constant coefficient simulation depends on estimate $\ddot{x}_{e}$ reproducing the instability behaviour caused by $\ddot{x}$ in equation (10). Figure 1 shows it to be extremely successful in this regard. In this figure the constant coefficient approximation was compared with actual ship motions computed by a time domain 'strip theory' program $[3,2]$. It represents a model ship in free heave oscillation due to an initial displacement (no incident waves, and other degrees of freedom restrained). Both simulations use the same time stepping algorithms with a time step size of approximately 0.0362 times the period of undamped natural oscillation.

The upper half of Figure 1 shows solutions with $\mu$ just above, just below, and somewhat lower than the stability threshold. The threshold differs slightly between the two methods but clearly the behaviour is the same. An initial "error" is deliberately introduced, resulting in the expected sawtooth oscillation implied by $\rho_{c}>1$. With the higher $\mu$ value the oscillations gradually grow with error in geometric progression, indicating instability, while with the intermediate value they gradually decay, indicating that the integration has just been stabilised. With the lower $\mu$ value we see that oscillations decay much more rapidly, that is, after only a few time steps. Of course without stabilisation $(\mu=1)$ the divergence would be extremely rapid. Interestingly, even when unstable the average force follows the stable form. The displacement graphs for all three $\mu$ values (not shown) are almost indistinguishable because the integrations smooth out the high frequency oscillations, although with the higher $\mu$ value the displacement graph eventually diverges.

Near the lower $\mu$ limit (lower half of Figure 1) the instability appeared as an oscillation at a frequency higher than the natural frequency but much lower than that of the sawtooth error near the upper $\mu$ limit. In the cases shown the undesired oscillations are decaying, so the solution is by definition stable, but the rate of decay for the lowest $\mu$ value shown is less than that of the true motion so the solution will never be accurate.

The integration algorithm, and its simulation by the constant coefficient 


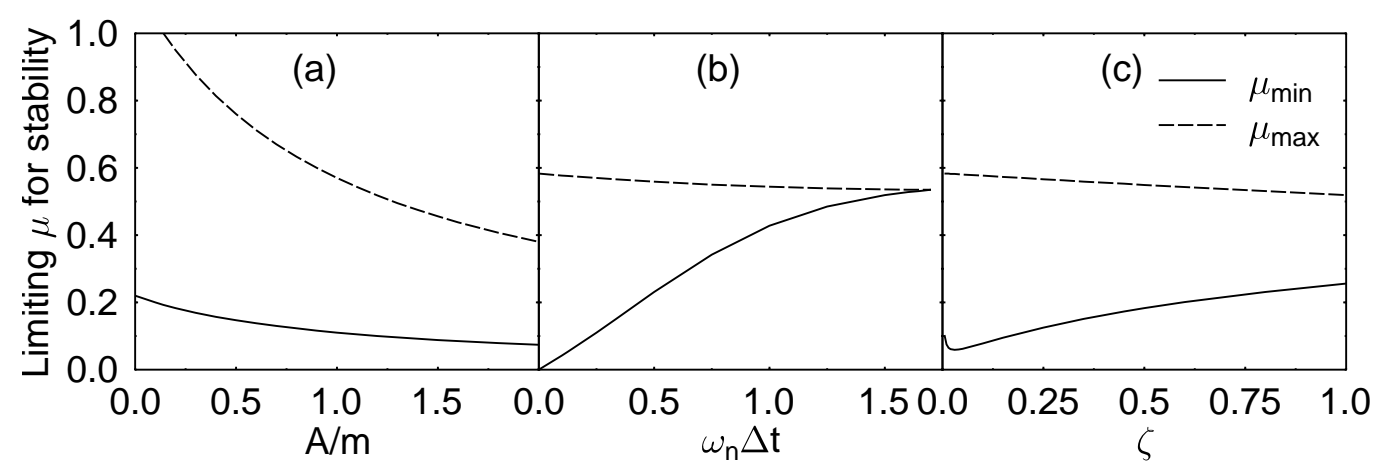

Figure 2: Limiting values of $\mu$ for stability at $\omega=\omega_{n}$ : (a) various $A / m$ $\left(\zeta=0.2, \omega_{n} \Delta t=0.25\right)$; (b) various $\Delta t(\zeta=0.2, A / m=1.0)$; (c) various $\zeta$ $\left(\omega_{n} \Delta t=0.25, A / m=1.0\right)$.

approximation, performed equally well with problems involving forces oscillation and multiple degrees of freedom.

\section{$5 \quad$ Limiting $\mu$ for stability}

Equation (5) shows that there are upper and lower limits of $\mu$ for which a stable solution may be obtained, and this section investigates these limits as determined empirically using the constant coefficient approximation described in the previous section. This does not necessarily imply accuracy of the solution, which will be discussed in the subsequent section.

I hypothesised that $\rho_{c}$ takes the form $\alpha A / m$ and $\rho_{e} \approx 1.5$, suggesting $c_{1} /\left(A / m+c_{2}\right)$ as the form of the $\mu$ limits, with $c_{1}$ and $c_{2}$ constants. Fitting such a form to the data of Figure 2(a) gives

$$
\frac{0.22}{A / m+1}<\mu<\frac{1.14}{A / m+1},
$$


(exact to three significant figures) which on comparison with (5) implies $\rho_{e}=1.48$ and $\rho_{c}=2.18 \mathrm{~A} / \mathrm{m}+0.69$, agreeing closely with the expected result. These relations are shown below to vary with damping ratio and time step size, but in general retain the same forms.

Of interest is that for small $A / m$ ( $<0.14$ in the above case) the problem is inherently stable, but this is rarely the case for ship motions. $A / m=1$ for a semicircle at infinite frequency in heave or sway motion, while ships typically have $A / m>1$ in heave, pitch and sway motions due to their higher beam to draught ratios. However, as stated above, for time domain calculations it is rarely practical to estimate the added mass before calculating motions so it is desirable that a reasonable range of $\mu$ exists. Figure 2(a) shows that this is the case.

Figure 2(b) shows that $\Delta t$ profoundly affects the limiting values of $\mu$. In particular, there is a maximum $\Delta t$ for which the method works; however, this corresponds to a time step size much larger than would ever be used in practice (3.7 time steps per natural period of oscillation). A more realistic maximum time step size of say $1 / 12$ th of the natural period, as might be required for moderate accuracy $\left(\omega_{n} \Delta t \approx 0.52\right)$, gives a good range of $\mu$.

Another remarkable observation is that $\mu_{\min } \rightarrow 0$ as $\Delta t \rightarrow 0$, meaning that only an infinitesimal proportion of the calculated force is required in this limit.

Figure 2(c) shows that the damping ratio over the subcritical range $(\zeta<$ 1) weakly affects stability in comparison with $A / m$ and $\Delta t$, although perhaps surprisingly, stability is reduced by increasing $\zeta$. Typically motion damping for ships in heave and pitch is in the range $\zeta=0.15-0.25$, sometimes artificially increased slightly beyond this with the use of ride control appendages. 


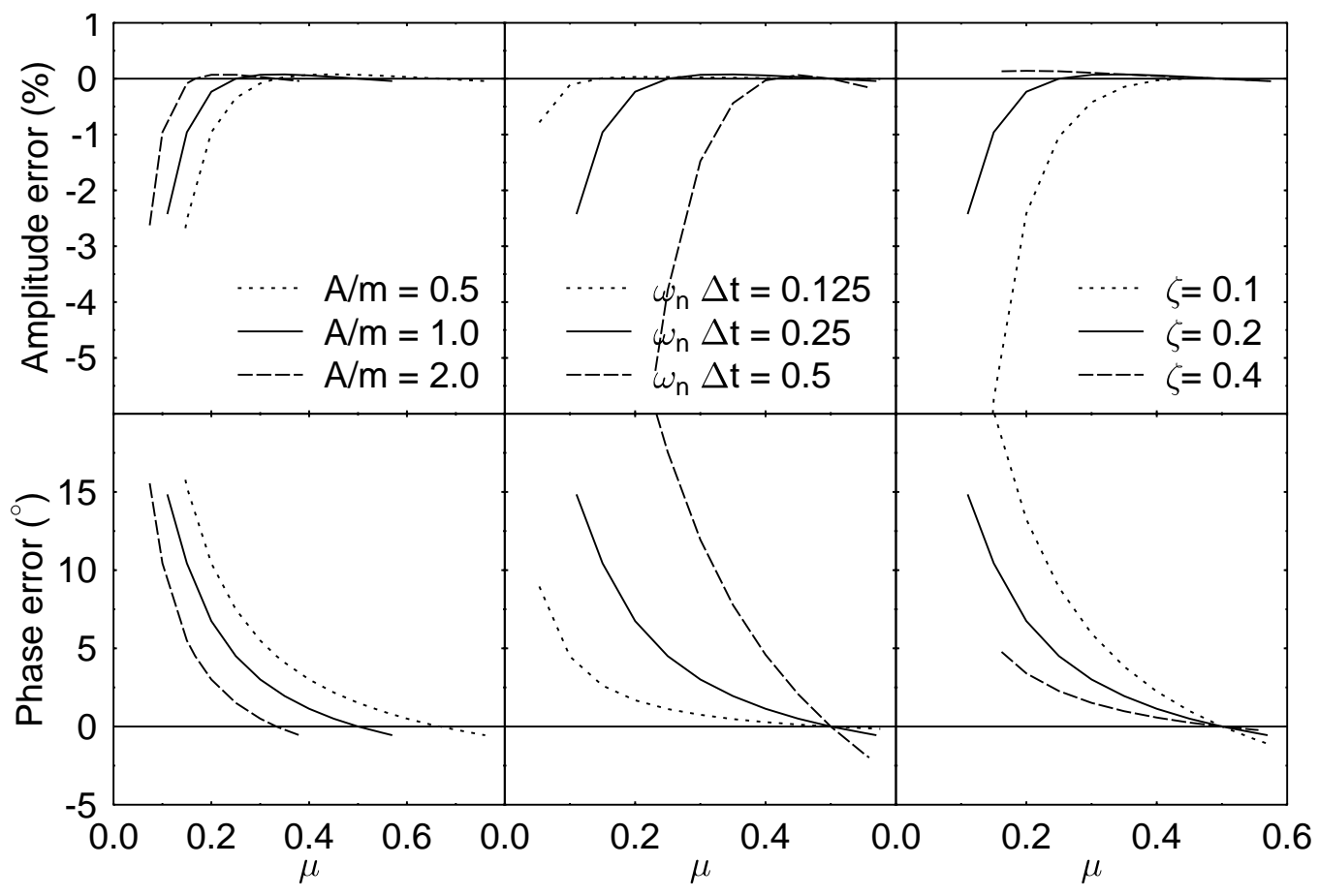

FiguRE 3: Error relative to stable solution at $\omega=\omega_{n}$ : (a) various $A / m$ ( $\left.\zeta=0.2, \omega_{n} \Delta t=0.25\right)$; (b) various $\Delta t=0.25(\zeta=0.2, A / m=1.0)$; (c) various $\zeta\left(\omega_{n} \Delta t=0.25, A / m=1.0\right)$.

\section{Influence of $\mu$ on accuracy}

Errors arise from both the numerical integration scheme, equations (7)-(9), and the stabilisation algorithm, equations (4) and (6). Errors presented below are the total unless otherwise stated.

Figure 3(a) shows how error varies over the full range of stable $\mu$ values for several $A / m$ ratios. The discretisation components of these errors are respectively $-0.32 \%$ and $15.9^{\circ}$ for amplitude and phase. Thus for higher 
values of $\mu$ the stabilisation errors are relatively small, and vanish for both amplitude and phase in all cases at $\mu=m /(A+m)$. The errors at the extremities of each curve are similar, thus error is essentially a function of $\mu$ relative to its limiting values for each problem, rather than to the absolute $\mu$. Apart from this indirect influence there is no evidence of any significant effect of $A / m$ on accuracy.

The time step size, as expected, profoundly affects accuracy (Figure 3(b)), but the stabilisation error remains approximately in proportion to the discretisation error. For $\mu$ near its upper stability limit the stabilisation error is negligible and at times even favourable, and the main concern for accuracy is at the lower end of the $\mu$ range.

Figure 3(c) shows the effect of damping ratio on the stabilisation error. Compared to the discretisation error $(-0.70 \%,-0.32 \%$ and $-0.15 \%$ in magnitude and $17.5^{\circ}, 15.9^{\circ}$ and $15.1^{\circ}$ for phase for $\zeta=0.1,0.2$ and 0.4 respectively) the influence of $\zeta$ on the stabilisation error is significantly stronger, although qualitatively the same, with higher damping ratios being more favourable.

The effect of frequency on the stabilisation error was also investigated. At high frequency the error becomes greater mainly because the time step size becomes large compared with the period of oscillation. However, the stabilisation component of the error is less than the discretisation error in the upper half of the stable $\mu$ range, and this holds for all frequencies.

Thus, in combination with the earlier observations, we conclude that it is primarily the $A / m$ ratio that determines the optimum $\mu$ for both accuracy and stability. 


\section{Conclusion}

A procedure was presented for stabilising explicit time stepping in initial value problems involving feedback in the highest derivative terms. The illustrative application was the unsteady motion of ships, in which the feedback arises from the added mass of the water surrounding the hull. The method involved elimination of the destabilising added mass effectively by estimating its value and moving it to the other side of the equation. In practice this was achieved simply by blending the acceleration calculated from the hydrodynamic force with an acceleration extrapolated from prior motions in suitable proportions designated by a parameter $\mu$. The instability present in ship motion computations was simulated by the use of a simplified approximation, both confirming the presumed origin of the problem and allowing easy testing of the stabilising algorithm over a wide range of scenarios.

The method was extremely successful over the range of parameter space into which normal ships could conceivably fall, providing both stability and reasonable accuracy. A wide range of $\mu$ gave stable solutions, hence the method is not dependent on accurate estimation of the added mass effect, making it quite robust.

Errors are introduced both by the time discretisation (present also in any stable problem) and by the stabilisation algorithm, and these two are generally similar. By choosing $\mu$ nearer the upper stability limit the stabilisation errors were minimised and small in comparison to the discretisation errors, while for lower values of $\mu$ the converse was true.

Although there may be significant coupling between the motions in the various degrees of freedom (for example between heave and pitch) experience shows that this does not translate into a coupling of the destabilising influences. There is only a very weak, if any, interaction between the stability of each degree of freedom, and the optimum stability coefficient for each can safely be determined independently, greatly simplifying the problem. 
Acknowledgements: This work was carried out with the support of the University of Tasmania and the Australian Maritime Engineering Cooperative Research Centre.

\section{References}

[1] R. F. Beck, Y. Cao, S. M. Scorpio, and W. W. Schultz. Nonlinear ship motion computatios using the desingularised method. In Twentieth Symposium on Naval Hydrodynamics, 1994. C252

[2] D. S. Holloway and M. R. Davis. Green function solutions for the transient motion of water sections. Journal of Ship Research, 46(2):99-120, June 2002. C252, C256

[3] D. S. Holloway and M. R. Davis. Ship motion computations using a high Froude number time domain strip theory. Journal of Ship Research, 50(1):15-30, March 2006. C256

[4] D. Kring and P. D. Sclavounos. Numerical stability analysis for time-domain ship motion simulations. Journal of Ship Research, 39(4):313-320, 1995. C252, C253 


\section{Author address}

1. D. S. Holloway, School of Engineering, University of Tasmania, Hobart, Australia.

mailto:Damien. Holloway@utas . edu . au 